\title{
Tyrosinase-Immobilized Biosensor Based on the Functionalized Hydroxyl Group-MWNT and Detection of Phenolic Compounds in Red Wines
}

\author{
Ji-Hoon Yang, Jae-Chan Lee, and Seong-Ho Choi \\ Department of Chemistry, BK 21 NanoBiosensor Research Team, Hannam University, Daejeon 305-811, South Korea \\ Correspondence should be addressed to Seong-Ho Choi, shchoi@hnu.kr
}

Received 24 July 2009; Accepted 31 August 2009

Recommended by Kourosh Kalantar-Zadeh

The tyrosinase-immobilized biosensor was developed with the hydroxyl group-functionalized multiwall carbon nanotube (MWNT) for phenol detection. The hydroxyl group-modified MWNT was modified to include poly(GVPB)-g-MWNT, or poly(HEMA), by a radiation-induced graft polymerization of glucosyl 4-vinylphenylboronate (GVPB) or 2-hydroxyethylmethacrylate (HEMA) on the surface of MWNT. The response of biosensor was in the range of 0.6-7.0 $\mathrm{mM}$ for concentration and in the range of $0.05-0.35 \mathrm{mM}$ for phenol in a phosphate buffer solution, respectively. Various parameters influencing biosensor performance have been optimized: for $\mathrm{pH}$, temperature, and the response to various phenolic compounds. The biosensor was then tested on phenolic compounds contained in three different commercial red wines.

Copyright (c) 2009 Ji-Hoon Yang et al. This is an open access article distributed under the Creative Commons Attribution License, which permits unrestricted use, distribution, and reproduction in any medium, provided the original work is properly cited.

\section{Introduction}

The surface functionalization of carbon nanotubes was performed to achieve the following two goals [1-4]. The first one was to render the nanotubes "soluble" in a liquid, and the second was to introduce functional groups onto the surface of carbon nanotubes. In either case, forming a stable dispersion was essential to the study of the chemistry of carbon nanotubes.

Radiation-induced graft polymerization (RIGP) is a useful technique for the introduction of functional groups into different polymer matrixes by using specially selected monomers. There have been several reports on RIGP of polar monomers onto polymer subtracts with hydrophobic properties in order to obtain hydrophilic properties for versatile applications [5-8]. In a previous paper [9], the MWNT was functionalized with various monomers by RIGP in an aqueous solution at a room temperature using $\gamma$-irradiation. The boric acid-functionalized MWNT was used as a supporting material for detecting glucose that has no enzymes.

In a previous paper [10], we also introduced the carboxylic acid group onto the surface of MWNT by RIGP of acrylic acid and methacrylic acid to increase the affinity of the enzymes. When we use the carboxylic acid-functionalized MWNT, the sensitivity of the biosensor is enhanced due to an increase in affinity between the MWNT surface and the enzymes. On the other hand, the hydroxyl group, $-\mathrm{OH}$, is well known as being one of the functional groups that has affinity to biomolecules [11-13]. The $-\mathrm{OH}$ group also has hydrophilic properties and consequently it can easily immobilize the enzyme onto the hydrophilic site by physical methods. However, little has been reported about the introduction of $-\mathrm{OH}$ groups onto CNT surfaces by grafting techniques.

Wines, particularly red wines, contain numerous biologically active compounds. The most important of which is poly-phenols. The nutritional importance of poly-phenols is attributed to their antioxidant properties. In particular, flavonoids and their related phenolic compounds, which are naturally found in red wines, are gaining interest [14]. Red wines have been reported to be more cardioprotective and they play a possible role in reducing thrombotic and anthropogenic processes. Poly-phenols also contribute substantially to the quality of wines as they affect the color, 


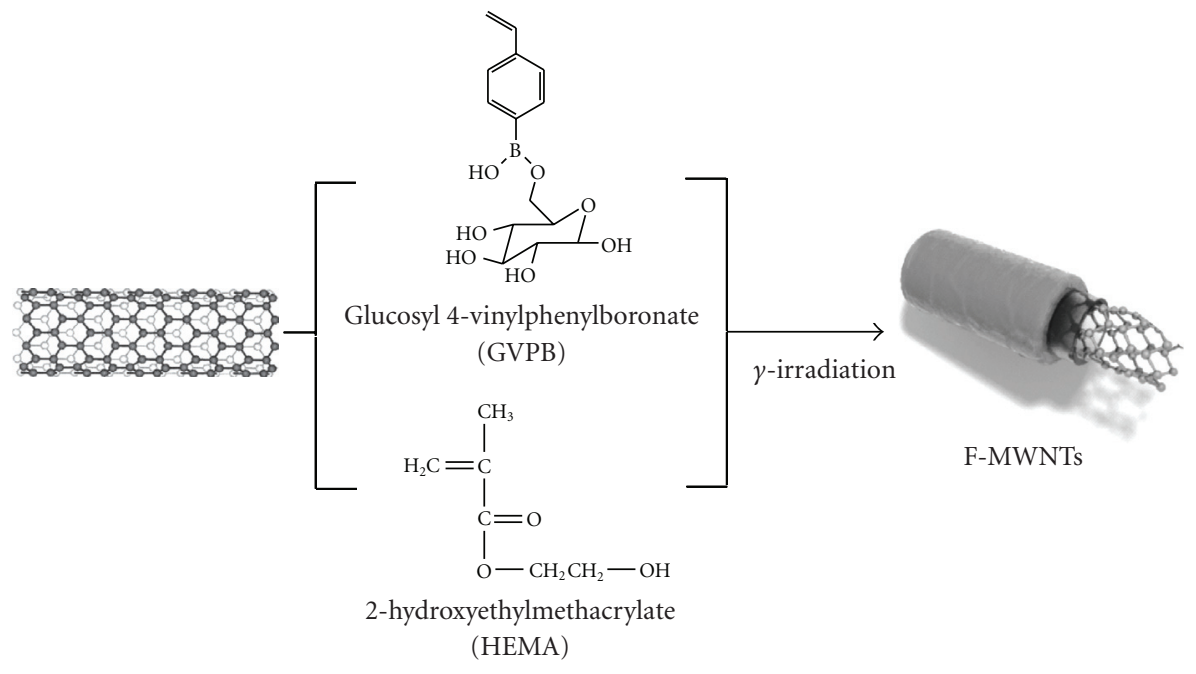

FIGURE 1: Radiation-induced graft polymerization of the vinyl monomers with hydroxyl group on the surface of MWNT.

flavor, stability, and aging behavior [15]. However, little has been reported on the total amount of phenolic compounds in red wine determined by electrochemical methods.

In this study, the tyrosinase-immobilized biosensor, based on $-\mathrm{OH}$ group functionalized MWNT was designed to detect or sense the phenolic compounds in red wines. The biosensor was fabricated on an ITO electrode via hand casting of chitosan solution with a combination of the hydroxyl group-functionalized MWNT and tyrosinase. The biosensor was evaluated on its efficiency in sensing phenols in a phosphate buffer solution. Various parameters influencing the response of biosensor were tested and optimized: $\mathrm{pH}$, temperature, and different phenolic compounds. The total concentration of phenolic compounds in commercial red wines was also measured.

\section{Experiment}

2.1. Reagents. Tyrosinase from mushroom (EC 1.14.18.1), $\alpha$-D-glucose, 2-hydroxyethylmethacrylate (HEMA), phenol, p-chresol, catechol, gallic acid, chitosan, and THF were purchased from the Aldrich-Sigma Chemical Co. (USA). 4Vinylphenylboronic acid was purchased from TCI Co., LTD (Japan). The ITO electrode (working area $0.7 \times 1.1 \mathrm{~cm}^{2}, 10 \Omega$ resistance) was purchased from Dasom RMS Co. (Korea). MWNT (CM-95) was supplied by Hanwha Nanotech Co., Ltd (Korea). The red wines used were Lindemans (brand name, made in Australia), Bordeaux (brand name, made in France), and Duchessa Lia (brand name, made in Italy). Solutions for the experiments were prepared with purified water in a Milli-Q puls water purification system (Millipore Co. Ltd., USA). The final resistance of water was $18.2 \mathrm{M} \Omega \mathrm{cm}^{-1}$ and gas was removed prior to each measurement.

2.2. Synthesis of Glucosyl 4-vinylphenylboronate (GVPB). Glucosyl 4-vinylphenylboronate (GVPB) was synthesized from a reaction of 4 -vinylphenylboronic acid $(3.4 \mathrm{mmol})$ and $\alpha$-D-glucose $(6.8 \mathrm{mmol})$ in $\mathrm{THF} / \mathrm{H}_{2} \mathrm{O}(50 / 50 \mathrm{~mL})$ at $60^{\circ} \mathrm{C}$ over 12 hours. The conversion yield was $\sim 68.9 \%$. Spectroscopic data of GVPB: ${ }^{1} \mathrm{H} \mathrm{NMR}\left(\mathrm{CDCl}_{3}, \mathrm{TMS}\right): \delta 8.2 \sim 7.5$ (t, 4H, phenyl), 7.3 (d, 1H, O-CH-OH), $6.8(\mathrm{~m}, 1 \mathrm{H}, \mathrm{C}=\mathrm{CH}-$ C), $5.9 \sim 5.4\left(\mathrm{~d}, 2 \mathrm{H}, \mathrm{CH}_{2}=\mathrm{C}\right), 1.6\left(\mathrm{~s}, 3 \mathrm{H}, \mathrm{O}-\mathrm{CH}_{2}-\mathrm{CH}-\mathrm{O}\right), 1.3$ (t, 2H, C-CH-C), 0.9 (t, 1H, C-CH-C). FT-IR (KBr): 3420 $(\mathrm{OH}), 1600(\mathrm{C}=\mathrm{C}), 1110(\mathrm{C}-\mathrm{O})$. LC-MASS: 310.12.

2.3. Synthesis of the Hydroxyl Group-Modified MWNT by RIGP. Figure 1 shows the RIGP of the vinyl monomers with $-\mathrm{OH}$ group functionalized on the surface of MWNT. The MWNT was purified to remove the catalyst and noncrystallized carbon impurities. In detail, the MWNT was treated with a mixture of $\mathrm{H}_{2} \mathrm{SO}_{4} / \mathrm{HNO}_{3}=3 / 1$ (vol-\%) and in the process the MWNT was cut into shorter segments. The purified and cut MWNT was used as the supporting material for grafting with GVPB and HEMA, respectively. The MWNT $(2.0 \mathrm{~g})$ was mixed with GVPB $(2.0 \mathrm{~g})$ or HEMA $(2.0 \mathrm{~g})$ in an aqueous solution $(20 \mathrm{~mL})$. Nitrogen was gas bubbled through the solution for 30 minutes in order to remove oxygen gas, and the solution was irradiated by $\gamma$-ray from Co-60 source under atmospheric pressure and ambient temperature. A total irradiation amount of $30 \mathrm{kGy}$ (a dose rate $\left.=1.0 \times 10^{4} \mathrm{~Gy} / \mathrm{h}\right)$ was used. The poly $(\mathrm{GVPB})-g$-MWNT and poly(HEMA)- $g$-MWNT were dried in vacuum oven at $50^{\circ} \mathrm{C}$.

2.4. Fabrication of the Enzyme Electrode Based on a Hydroxyl Group-Modified MWNT. The mixed solution containing chitosan (3.2 mg) as the binder, tyrosinase (25,000 unit, $0.3 \mathrm{mg}$ ), and the hydroxyl group-functionalized MWNT $(3.2 \mathrm{mg})$ as the support in acetic acid solution $(0.01 \mathrm{~mL})$ was prepared. A precleaned ITO electrode by a hand-cast method was coated on the surface with the mixed solution $(9.0 \mu \mathrm{L})$. The prepared enzyme electrode was maintained at $4^{\circ} \mathrm{C}$ until used.

2.5. Instrumentation. Electrochemical measurements were performed with a Potentiostat/Gavanostat model 283 (Ametek PAR, USA) or CV-50w voltametry (Bioanalytical 


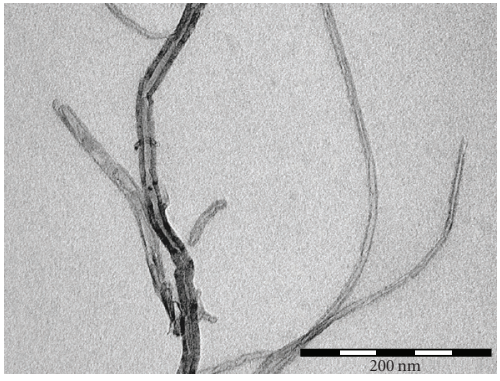

(a)

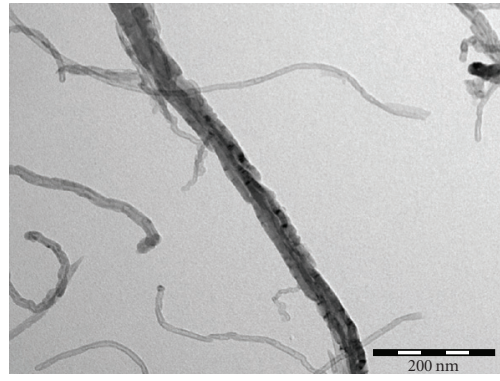

(b)

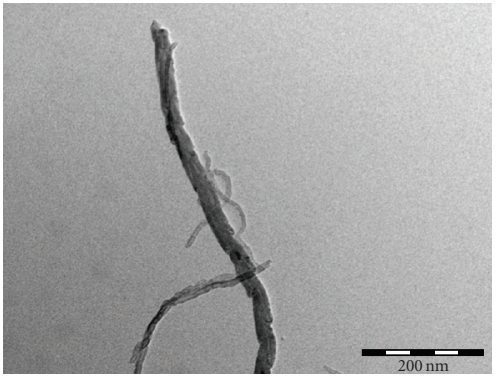

(c)

Figure 2: TEM images of the purified MWNT (a), poly(GVPB)- $g$-MWNT (b), and poly(HEMA)- $g$-MWNT (c).

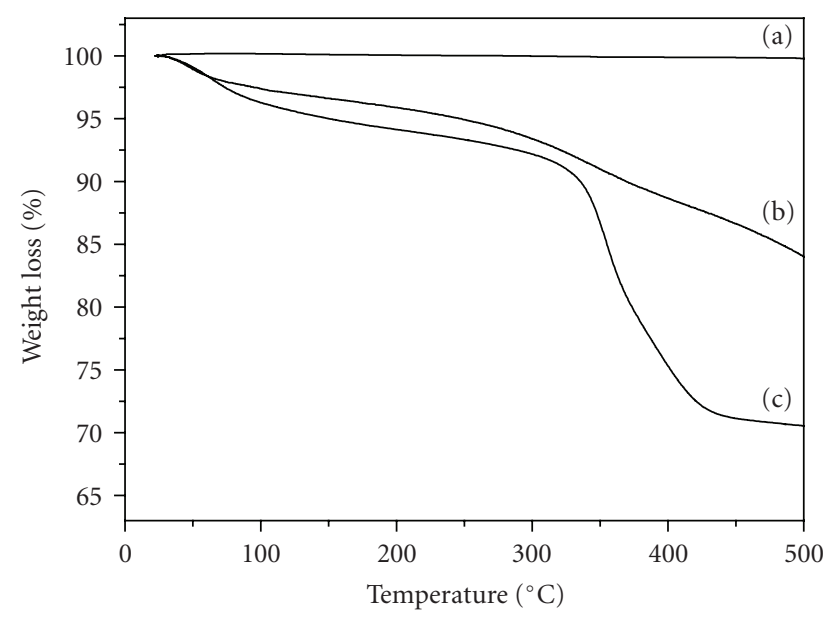

FIGURe 3: TGA curves of the purified MWNT (a), poly(GVPB)-gMWNT (b), and poly(HEMA)-g-MWNT (c).

Systems, Inc. USA). All experiments were carried out with a conventional three-electrode system. The working electrode, ITO, was coated with the poly(GVPB)-g-MWNT or poly(HEMA)- $g$-MWNT, the counter electrode was platinum wire, and the reference electrode was an $\mathrm{Ag} / \mathrm{AgCl}$ (sat'd $\mathrm{KCl}$ ). The surface morphology of the samples was determined using electron scanning microscopy (SEM, S-3000N, Hitachi Science System Ltd., Japan), by HR-TEM (JEOL, JEM-2010, USA) for measuring the functionalized, and atomic force microscopy (AFM, PICO station, Germany). The thermal gravimetric analysis (TGA) was conducted on a Scinco TGA S-1000 (Seoul, Korea) under $\mathrm{N}_{2}$ flow from $25^{\circ} \mathrm{C}$ to $700^{\circ} \mathrm{C}$ at a heating rate of $20^{\circ} \mathrm{C} / \mathrm{min}$.

\section{Results and Discussion}

3.1. Fabrication and Characterization of Enzyme Electrodes Based on the Hydroxyl Group-Functionalized MWNT. The functionalization of MWNT is one of the most active fields in nanotube research providing an effective tool to broaden the electrochemical application spectrum of MWNT. We functionalized MWNT by a radiation-induced graft poly- merization of GVPB and HEMA monomers in an aqueous solution. Figure 2 shows the TEM images of the (a) purified MWNT, (b) poly(GVPB)- $g$-MWNT, and (c) poly(HEMA)$g$-MWNT. The diameter of the purified MWNT was $11 \mathrm{~nm}$, as shown in Figure 2(a), whereas after the RIGP, the diameter was increased to $42 \mathrm{~nm}$ of poly (GVPB) $-g$-MWNT and $29 \mathrm{~nm}$ of poly(HEMA)- $g$-MWNT, as shown in Figures 2(b)-2(c). The thickness of poly(GVPB)- $g$-MWNT and poly(HEMA)$g$-MWNT with a tubular-type morphology was $15 \mathrm{~nm}$ and $8.5 \mathrm{~nm}$, respectively, calculated from TEM. An increase in thickness of poly(GVPB)-g-MWNT is due to the size of the glucosyl group of the grafted poly(GVPB) which is bigger than that of the hydroxyl ethyl group of the grafted poly(HEMA). The formation of tubular-type morphology is due to the following reasons: in order to perform the radiation grafting, we used vinyl monomers because they have the hydrophilic site of hydroxyl group $(-\mathrm{OH})$ and hydrophobic site of vinyl group in a reactive solution (Figure 1). The vinyl group in the monomer is faced to the surface of the MWNT because of a hydrophobic-hydrophobic interaction, while the hydroxyl group of monomers is faced in an aqueous solution because of a hydrophilic-hydrophilic interaction. When the $\gamma$-ray is applied, the radical polymerization of vinyl monomers on the surface of MWNT occurs. As a result, we can obtain tubular functionalized MWNT through a one-step reaction. We can immobilize the enzyme to the functional group of the MWNT surface by physical adsorption, and we can also use the MWNT as an electron transfer material in order to increase biosensor sensitivity.

Figure 3 shows the TGA curves of (a) the purified MWNT, (b) poly(GVPB)- $g$-MWNT, and (c) poly(HEMA)$g$-MWNT prepared by RIGP. The 1st weight loss (\%) from $50^{\circ} \mathrm{C}$ to $300^{\circ} \mathrm{C}$ for a vinyl polymer-graft MWNT appeared to be due to a loss of moisture because of the hydrophilic properties of the grafted vinyl polymer. The 2 nd weight loss (\%) appeared in the range of $300-500^{\circ} \mathrm{C}$ due to the grafted vinyl polymer weight loss. The results show that the graft yields (\%) were in the range of 25-30\% after the RIGP of vinyl monomers.

Figures 4 and 5 show SEM and AFM-3D images of the tyrosinase-immobilized biosensor based on the functionalized MWNT: (a) Surface of the ITO electrode, (b) 


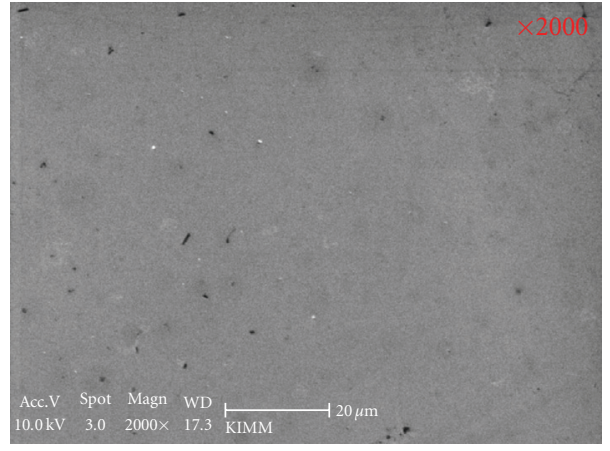

(a)

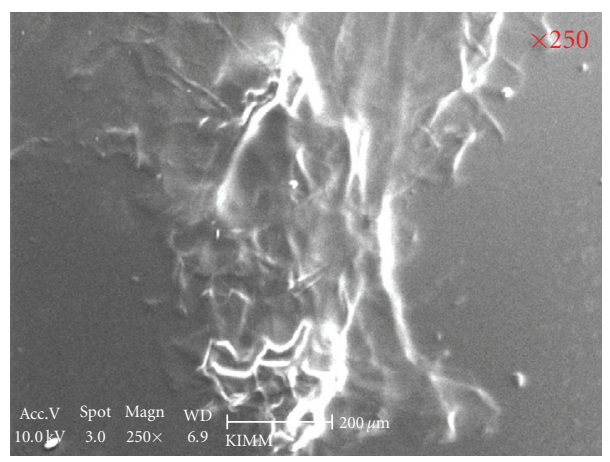

(c)

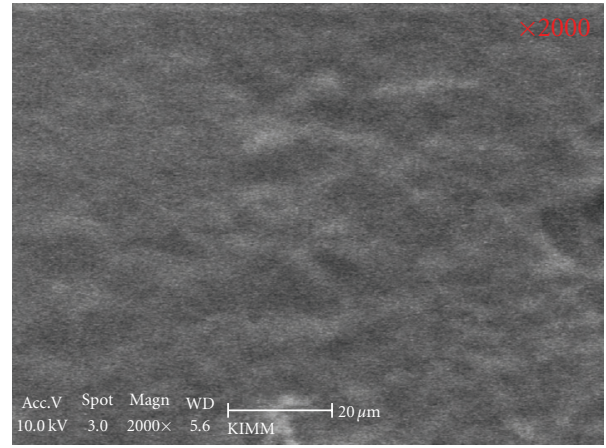

(b)

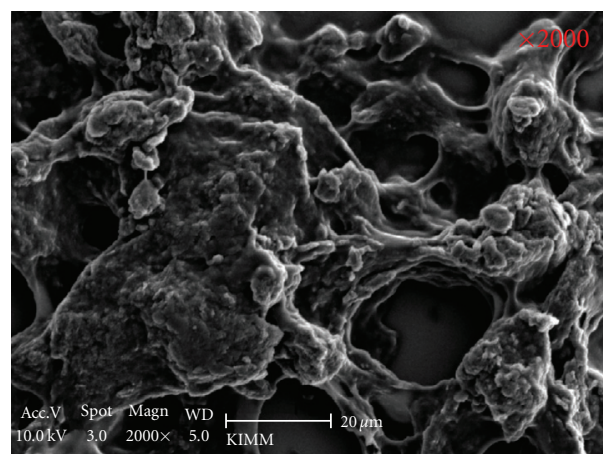

(d)

FIGURE 4: SEM images of the surface of ITO glass (a), chitosan-coated ITO (b), tyrosinase-immobilized chitosan-ITO electrode (c), and tyrosinase-immobilized biosensor based on poly(GVPB)- $g$-MWNT (d).

chitosan-coated ITO, (c) tyrosinase-immobilized chitosanITO electrode, and (d) a tyrosinase-immobilized biosensor based on poly(GVPB)- $g$-MWNT. The surface of the ITO is a flat form (Figure 4(a)), whereas the film of chitosan as the binder appeared flat but amorphous so that it resembles a typical polymer form, as shown in Figure 4(b). In contrast, the composite film with poly (GVPB)- $g$-MWNT has irregular curved peaks, as shown in Figure 4(c). The glucosyl group of poly(GVPB)-g-MWNT in a composite film can be immobilized by the tyrosinase and as a result, the sensitivity can be increased for ethanol. After the immobilization of tyrosinase on the MWNT-based composite film, the morphology of the composite film is changed, as shown in Figure 4(d). As a result, we successfully immobilized the tyrosinase using a hydroxyl group-modified MWNT. In the AFM-3D image, after the immobilization of tyrosinase onto the MWNTbased composite film, the surface morphology has a very smooth form, as shown in Figure 5(d), compared to that of the composite film in Figure 5(c) due to the morphology of the immobilized tyrosinase.

In order to determine the biosensor sensitivity, we designed four biosensors with and without the functionalized MWNT. Figure 6 shows the cyclic voltammograms of $0.5 \mathrm{mM}$ phenol in $50 \mathrm{mM}$ phosphate buffer solution using (a) an ITO electrode with tyrosinase, (b) an electrode with pure MWNT-tyrosinase, and (c) the biosensor based on poly(GVPB)-g-MWNT. The potential was scanned between
-1.0 and 0.75 versus $\mathrm{Ag} / \mathrm{AgCl}$ at a scan rate of $100 \mathrm{mVs}^{-1}$. The oxidation peak at $-0.3 \mathrm{~V}$ and low-reduction peak at $0.3 \mathrm{~V}$ on the biosensor are based on poly(GVPB)- $g$-MWNT, as shown in Figures 6(c) and 7. This peak represents the production of catechol or $o$-quinone from the enzymatic reaction in phosphate solution. In other words, tyrosinase is a copper-containing monooxygenase enzyme that catalyses conversion of phenolic substrates to catechol and then $o$ quinone as follows:

$$
\begin{aligned}
& \text { phenol }+ \text { tyrosinase }\left(\mathrm{O}_{2}\right) \longrightarrow \text { catechol, } \\
& \text { catecohol }+ \text { tyrosinase }\left(\mathrm{O}_{2}\right) \longrightarrow \text { o-quinone }+\mathrm{H}_{2} \mathrm{O}, \\
& \text { o-quinone }+\mathrm{H}^{+}+2 \mathrm{e}^{-} \longrightarrow \text { catechol. }
\end{aligned}
$$

3.2. Optimization of the Prepared Biosensor. Selectivity is a very important factor in the biosensor; so four compounds ( phenol, $p$-cresol, catechol, and gallic acid) were detected based on poly(GVPB)-g-MWNT in a $50 \mathrm{mM}$ phosphate buffer solution $(\mathrm{pH}=7.0)$. In the $p$-cresol and gallic acid solution, there are no redox peaks using the biosensor, as shown in Figure 7(b). Serra et al. [16] reported that the different sensitivity was observed for phenolic compounds with different positions in an enzymatic reaction. The metaposition phenol ( $m$-cresol) appears as the high response sensitivity more than ortho- and paraposition phenols in an enzymatic reaction due to a high affinity with tyrosinase. 


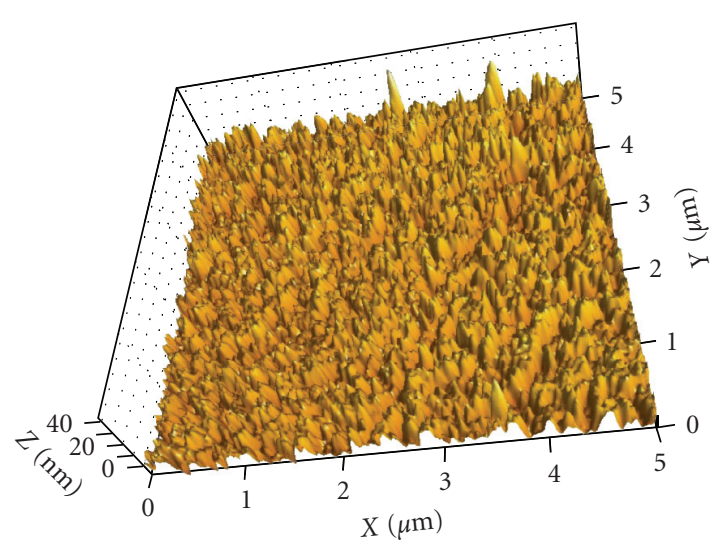

(a)

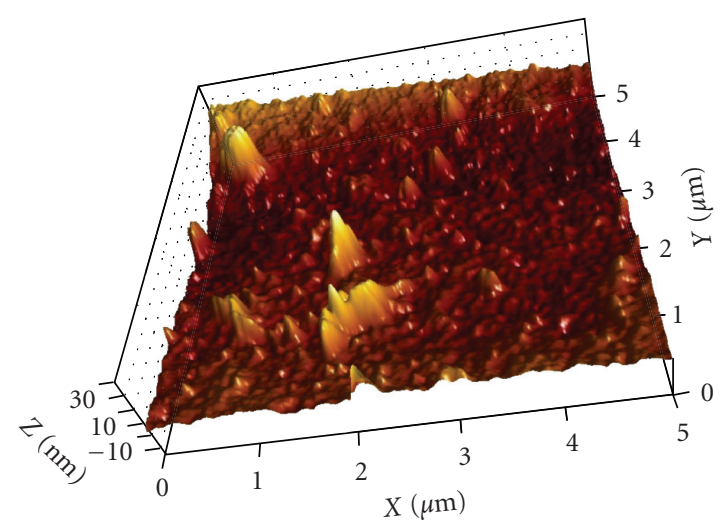

(c)

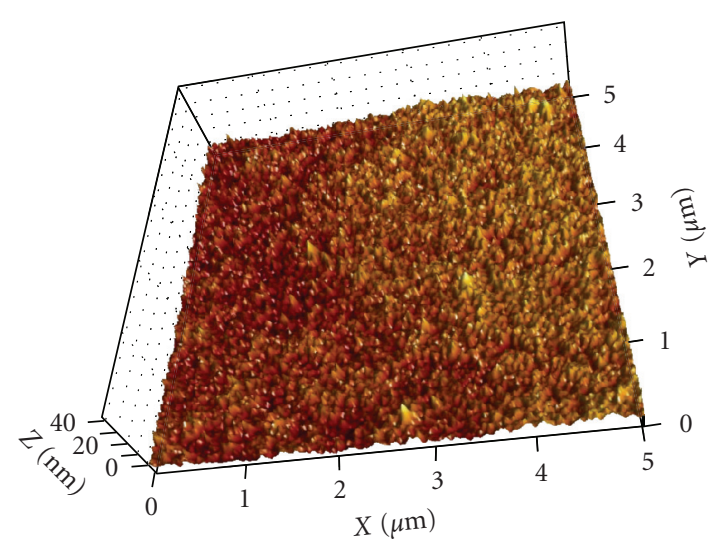

(b)

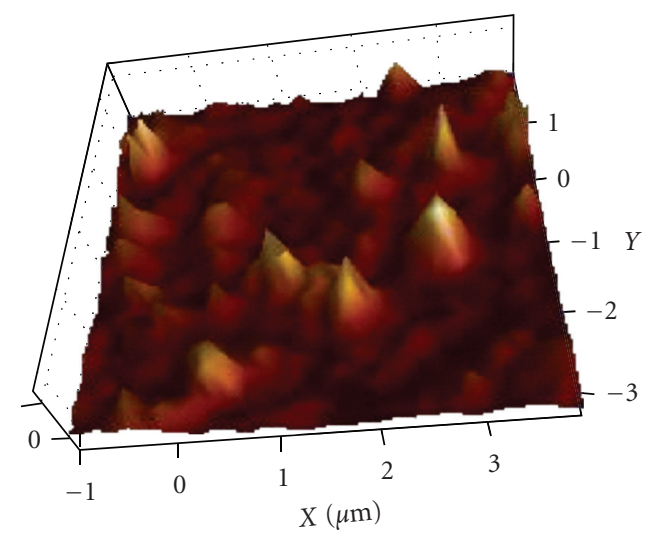

(d)

FIGURE 5: AFM 3-D images ( $5 \mu \mathrm{m}$ scale) of the surface of ITO glass (a), chitosan-coated ITO (b), tyrosinase-immobilized chitosan-ITO electrode (c), and tyrosinase-immobilized biosensor based on poly(GVPB)-g-MWNT (d).

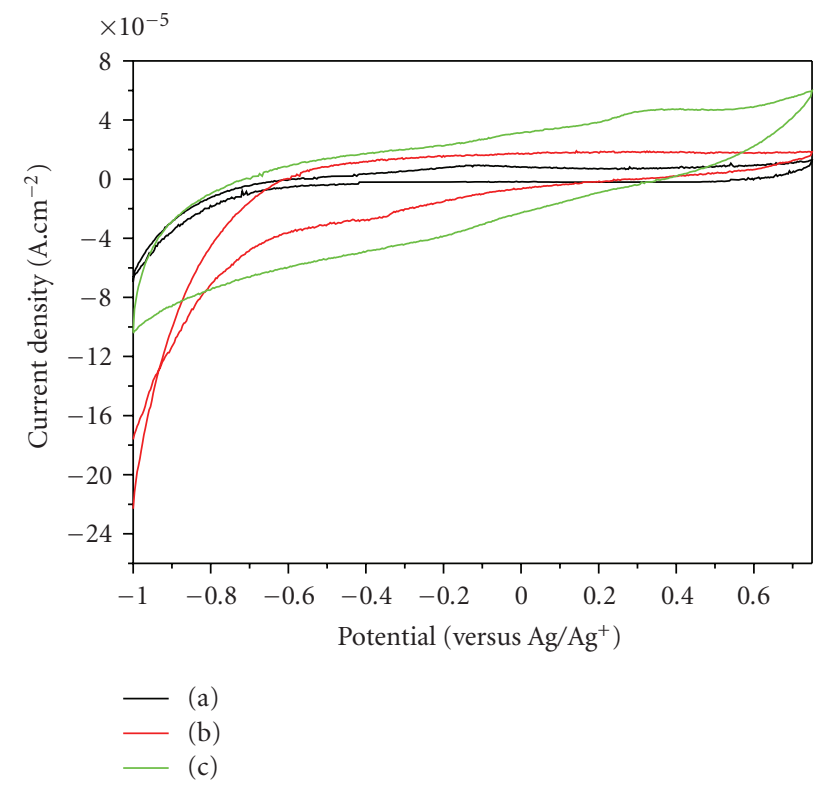

FIgURE 6: Cyclic voltammetry responses of $1.0 \mathrm{mM}$ phenol using tyrosinase-immobilized ITO electrode (a), tyrosinase-immobilized electrode based on purified MWNT (b), and tyrosinase-immobilized biosensor based on GVPB- $g$-MWNT (c) in 50 mM phosphate buffer solution with a scan rate $100 \mathrm{mV} / \mathrm{s}$. 


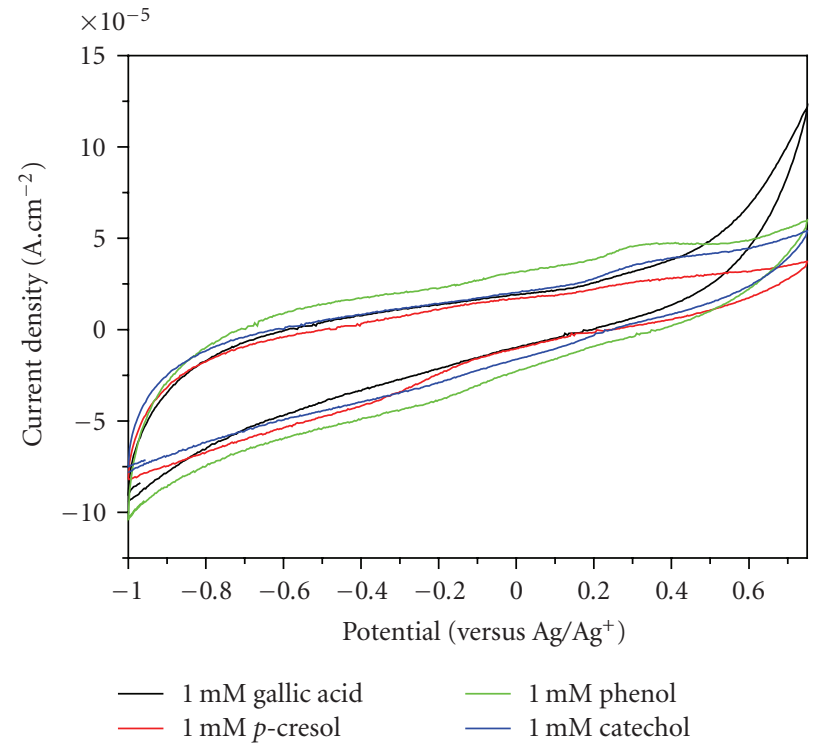

(a)
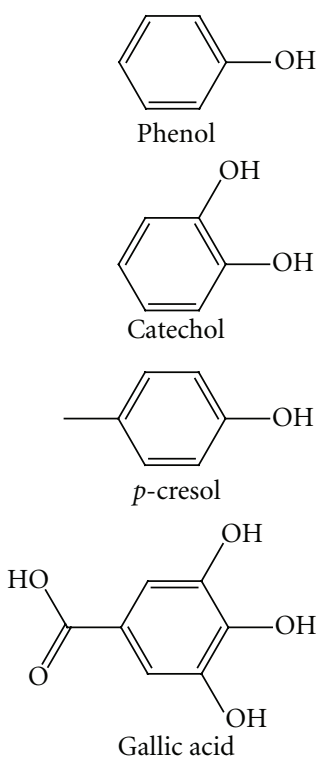

(b)

FIgURE 7: Cyclic voltammetry responses of tyrosinase-immobilized biosensor based on poly(GVPB)- $g$-MWNT in $1.0 \mathrm{mM}$ phenolic compounds. Scan rate is $100 \mathrm{mVs}^{-1}$. Phosphate buffer $(\mathrm{pH}=7.0)$ is at room temperature.

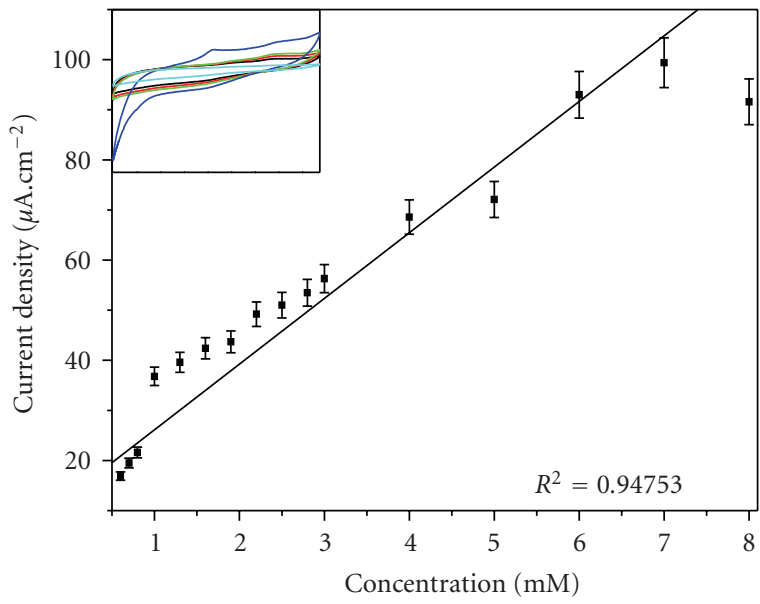

(a)

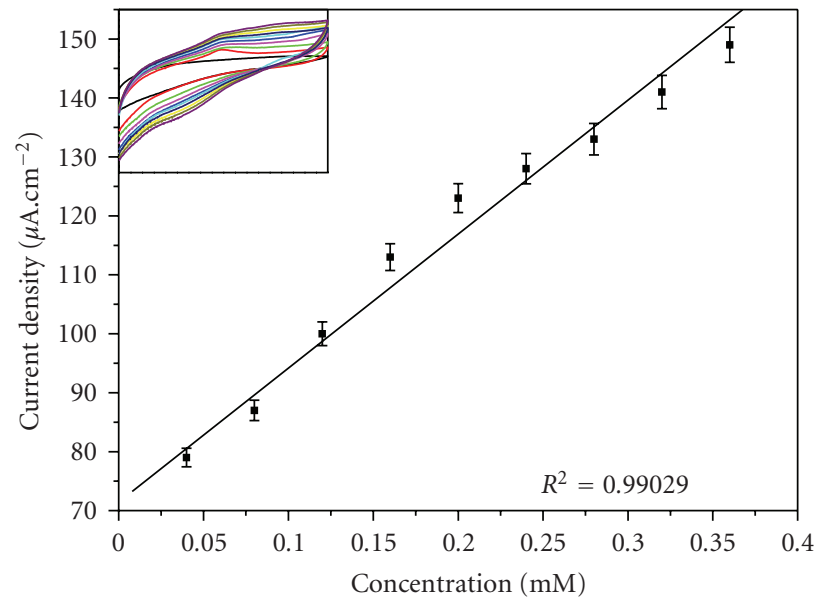

(b)

FIGURE 8: Calibration curves for phenol concentration on the tyrosinase-immobilized biosensor based on poly(GVPB)- $g$-MWNT (a) and poly(HEMA)- $g$-MWNT (b). Scan rate is $100 \mathrm{mVs}^{-1}$. Phosphate buffer $(\mathrm{pH}=7.0)$ is at room temperature.

Also shown in Figure 7, the high response peak of phenol appears more than that of $p$-cresol, catecohol, and gallic acid because of its enzymatic specificity. For catechol, the oxidation peak is at $-0.3 \mathrm{~V}$ and the reduction peak is $0.3 \mathrm{~V}$, and the high response peak appears to be more than that of $p$-cresol. Tsai et al. [17] have reported that $p$-catechol appears to have a greater sensitivity response than that of dopamine and epinephrine.

The electrochemical biosensing of phenol was performed under optimal experimental conditions. Figure 8 shows the cyclic voltammograms of phenol on biosensor based on (a)
poly(GVPB)- $g$-MWNT, and (b) poly(HEMA)- $g$-MWNT in $50 \mathrm{mM}$ phosphate buffer solution $(\mathrm{pH}=7.0)$ as function of phenol concentration. When using the poly(GVPB)-gMWNT biosensor, the sensing range of phenol was from 0.6 to $7.0 \mathrm{mM}$ concentration, as shown in Figure 8(a), whereas the sensing range of the biosensor based on poly(HEMA)- $g$-MWNT for phenol was in the range of $0.05-0.35 \mathrm{mM}$ concentration as shown in Figure 8(b). The sensitivity and apparent Michaelies-Menten constant $\left(\mathrm{K}_{\mathrm{M}^{\text {app }}}\right)$ of the biosensor based on poly (GVPB)-g-MWNT was $0.13 \mathrm{~A}$ $\left(\mathrm{molL}^{-1}\right)^{-1}$ and $54.9 \mathrm{mmolL}^{-1}$, respectively. The sensitivity 


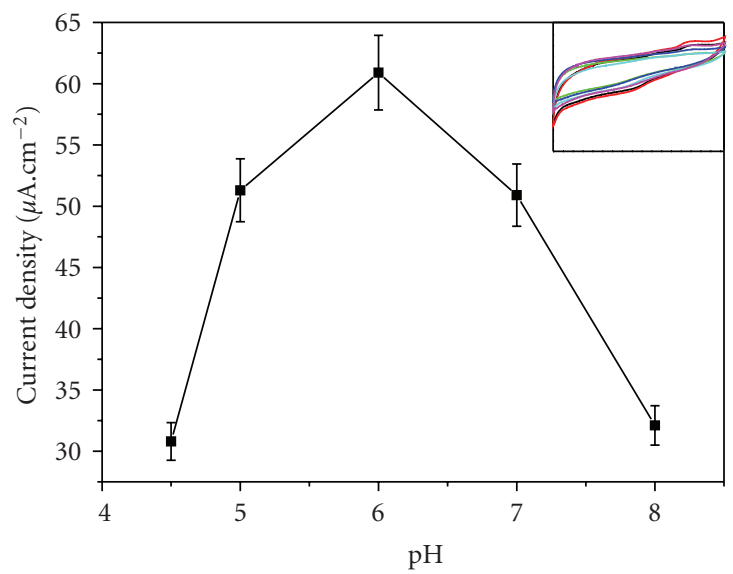

(a)

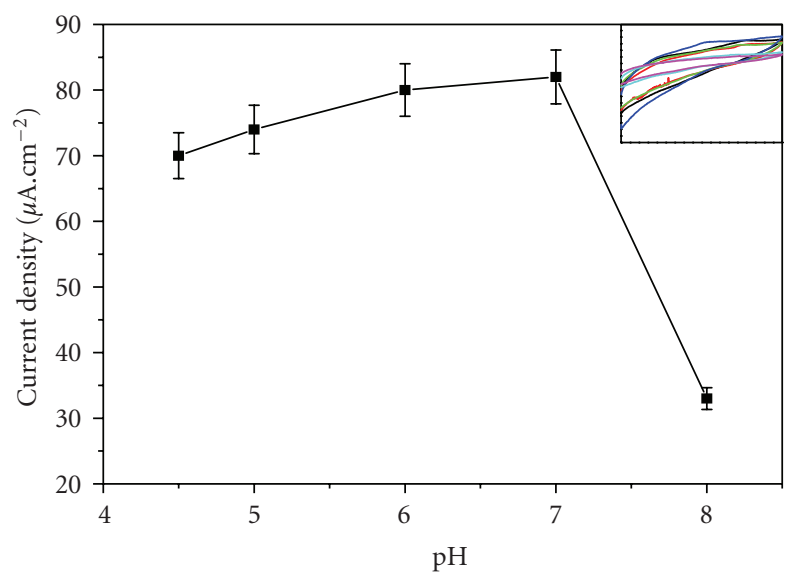

(b)

FIGURE 9: Effect of $\mathrm{pH}$ on tyrosinase-immobilized biosensor based on poly(GVPB)- $g$-MWNT (a) and poly(HEMA)- $g$-MWNT (b) on $1.0 \mathrm{mM}$ phenol.

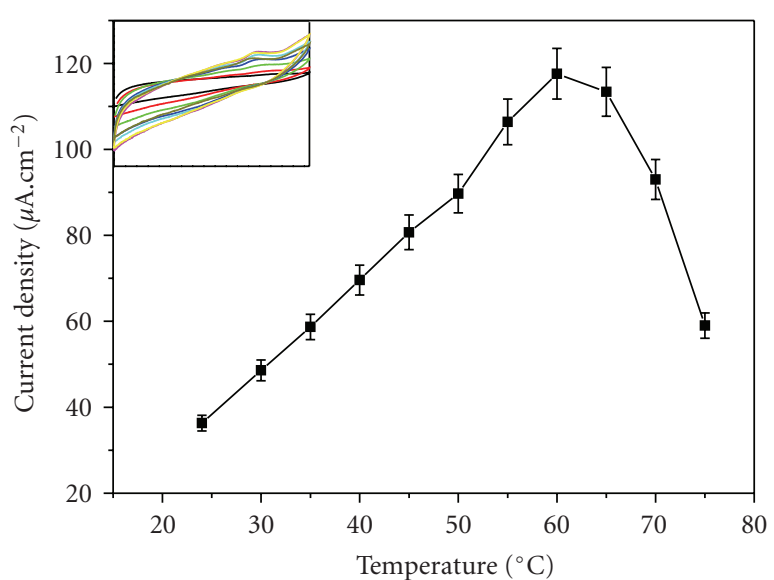

(a)

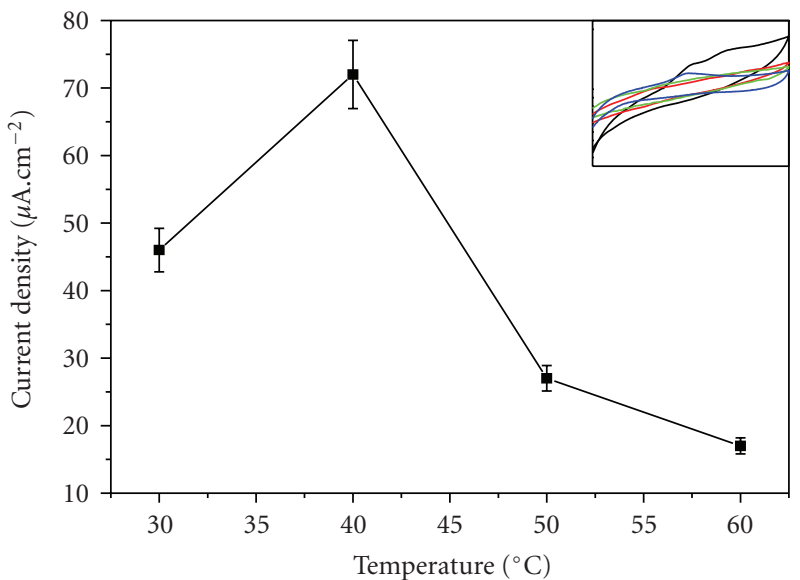

(b)

FigURE 10: Effect of Temperature on tyrosinase-immobilized biosensor based on poly(GVPB)-g-MWNT (a) and poly(HEMA)- $g$-MWNT (b) on $1.0 \mathrm{mM}$ phenol.

and $\mathrm{K}_{\mathrm{M}^{\text {app }}}$ of biosensor prepared by poly(HEMA)- $g$-MWNT was estimated to be $0.21 \mathrm{~A}\left(\mathrm{molL}^{-1}\right)^{-1}$ and $0.30 \mathrm{mmolL}^{-1}$, respectively. The $\mathrm{K}_{\mathrm{M}^{\mathrm{app}}}$ values were lower than those reported for free enzymes in a solution, which was estimated to be at $700 \mu \mathrm{molL}^{-1}$ as a substrate [18]. Such low $\mathrm{K}_{\mathrm{M}^{\text {app }}}$ values may be ascribed to the high concentration of tyrosinase on the biosensor. Additionally, the functionalized MWNT in a composite film may have increased the enzyme catalytic sites access to substrate molecules.

$\mathrm{pH}$ also plays an important role in the sensing efficiency of the biosensor. Figure 9 shows cyclic voltammograms of $0.5 \mathrm{mM}$ phenol on the biosensor based on (a) poly(GVPB)$g$-MWNT, (b) poly(HEMA)- $g$-MWNT in $50 \mathrm{mM}$ phosphate buffer solution as a function of $\mathrm{pH}$. The sensing efficiency is increased with increasing $\mathrm{pH}$ value from 4.0 to 6.0 and then decreased with increasing pH value as shown in Figure 9(a). This means that at a low $\mathrm{pH}$ value, the sensing efficiency of biosensor is due to enzyme activity. In contrast with low $\mathrm{pH}$ value, when using $\mathrm{pH}>6.0$, the sensing efficiency is rapidly decreased, while the sensitivity is similar in the range of $\mathrm{pH}=6$ and 7 on tyrosinase-immobilized biosensor based on poly(HEMA)- $g$-MWNT as shown in Figure 9(b).

The effect of temperature on the amperometric response of phenol was also studied. Before amperometric detection, the biosensor was immersed in a buffer solution at a given temperature for 10 minutes, and the CV was recorded. As shown in Figure 10(a), the sensitivity is increased with increasing temperature from 20 to $60^{\circ} \mathrm{C}$ and then sharply decreased when the temperature is increased further. Maximum sensitivity is attained at $60^{\circ} \mathrm{C}$. At high temperatures, the sensitivity of the biosensor for phenol is sharply decreased due to the partial denaturation of the immobilized tyrosinase. Although the sensitivity was the greatest at $60^{\circ} \mathrm{C}$, the study was conducted at room temperature because, first, 
TABLE 1: Total concentration of phenolic compounds for commercial wines on tryanosinase-immobilized biosensor based on poly(GVPB)$g-\mathrm{MWNT}^{(\mathrm{a})}$.

\begin{tabular}{lccc}
\hline Brand & Country & Current density (A) & Phenolic compounds (mg/L) \\
\hline Lindemans & Australia & $1.97 \times 10^{-5}$ & 68.50 \\
Bordeaux & France & $4.70 \times 10^{-5}$ & 218.5 \\
Duchessa Lia & Italy & $9.53 \times 10^{-5}$ & 655.0 \\
\hline
\end{tabular}

(a) The sensing was performed in $2.0 \mathrm{~mL}$ commercial wine.

most enzymes could be easily denaturated at high temperatures and, second, the practical application requires simple experimental procedures and long lifetime of the biosensor. The glucosyl group is enhanced by the temperature stability for the enzyme. On the other hand, maximum sensitivity is obtained at $40^{\circ} \mathrm{C}$ on a biosensor based on poly(HEMA)-gMWNT, as shown in Figure 10(b).

3.3. Total Concentration of the Phenolic Compounds in Commercial Red Wines. Three red wines, Lindemans, made in Australia, Bordeaux, made in France, and Duchessa Lia, made in Italy, were used in the analysis of phenolic amounts in wines, as described in Table 1. The sensing using the biosensor based on poly(GVPB)-g-MWNT was performed in $2.0 \mathrm{~mL}$ red wines directly. The data obtained from red wines is compared with calibration curves obtained from phenol solution as shown in Figure 8(a).

\section{Conclusion}

In this study, the tyrosinase-immobilized biosensor based on poly(GVPB)- $g$-MWNT and poly(HEMA)- $g$-MWNT was prepared by radiation-induced graft polymerization. The sensing range of the biosensor for phenol was in the range of $0.6 \sim 7.0 \mathrm{mM}$ and $0.05 \sim 0.35 \mathrm{mM}$ concentration, respectively. The biosensor was optimized for $\mathrm{pH}$, temperature, and other phenolic compounds in the determination of phenolics in commercial red wines. As a result, the amounts of phenolic compounds in commercial red wines are in the range of $68.50 \sim 655.0 \mathrm{mg} / \mathrm{L}$ which were calculated from a calibration curve of phenol on a biosensor based on poly(GVPB)- $g$ MWNT, as shown in Figure 8(a). Ten-times the amount of phenolic compounds in the Duchessa Lia was contained and compared to that of Lindemans. The conclusion is that high amounts of phenolic compounds in Duchessa Lia are responsible for the bitter taste of the red wine.

\section{Acknowledgment}

This study is supported by the Nano R\&D program via the Korea Science and Engineering Foundation funded by the Ministry of Science and Technology.

\section{References}

[1] A. Aziz, S. J. Park, S. Y. Jon, and H. S. Yang, "Amperometric immunosensing using an indium tin oxide electrode modified with multi-walled carbon nanotube and poly(ethylene glycol)silane copolymer," Chemical Communications, no. 25, pp. 2610-2612, 2007.
[2] C. Hu, C. Yang, and S. Hu, "Hydrophobic adsorption of surfactants on water-soluble carbon nanotubes: a simple approach to improve sensitivity and antifouling capacity of carbon nanotubes-based electrochemical sensors," Electrochemistry Communications, vol. 9, no. 1, pp. 128-134, 2007.

[3] C.-Y. Hong, Y.-Z. You, and C.-Y. Pan, "A new approach to functionalize multi-walled carbon nanotubes by the use of functional polymers," Polymer, vol. 47, no. 12, pp. 4300-4309, 2006.

[4] J. G. Deng, X. B. Ding, Y. X. Peng, and A. S. C. Chan, "Carbon nanotube-polyaniline hybrid materials," European Polymer Journal, vol. 38, no. 12, pp. 2497-2501, 2002.

[5] S.-H. Choi and Y. C. Nho, "Adsorption of $\mathrm{UO}^{2+}{ }_{2}$ by polyethylene adsorbents with amidoxime, carboxyl, and amidoxime/carboxyl group," Radiation Physics and Chemistry, vol. 57, no. 2, pp. 187-193, 2000.

[6] S.-H. Choi, S.-Y. Park, and Y. C. Nho, "Electrochemical properties of polyethylene membrane modified with carboxylic acid group," Radiation Physics and Chemistry, vol. 57, no. 2, pp. 179-186, 2000.

[7] S.-H. Choi and Y. C. Nho, "Radiation-induced graft copolymerization of binary monomer mixture containing acrylonitrile onto polyethylene films," Radiation Physics and Chemistry, vol. 58, no. 2, pp. 157-168, 2000.

[8] S.-H. Choi, K.-P. Lee, J.-G. Lee, and Y. C. Nho, "Graft copolymer-metal complexes obtained by radiation grafting on polyethylene film," Journal of Applied Polymer Science, vol. 77, no. 3, pp. 500-508, 2000.

[9] D.-S. Yang, D.-J. Jung, and S.-H. Choi, submitted toRadiation Physics and Chemistry.

[10] M.-H. Piao, D.-S. Yang, K.-R. Yoon, S.-H. Lee, and S.-H. Choi, "Development of an Electrogenerated Chemiluminescence Biosensor using Carboxylic acid-functionalized MWCNT and Au Nanoparticles," Sensors, vol. 9, pp. 1662-1677, 2009.

[11] Y. H. Yun, Z. G. Dong, V. Shanov, et al., "Nanotube electrodes and biosensors," Nano Today, vol. 2, no. 6, pp. 30-37, 2007.

[12] F. R. R. Teles and L. P. Fonseca, "Applications of polymers for biomolecule immobilization in electrochemical biosensors," Materials Science and Engineering C, vol. 28, no. 8, pp. 15301543, 2008.

[13] S. Akgöl, Y. Kaçar, A. Denizli, and M. Arca, "Hydrolysis of sucrose by invertase immobilized onto novel magnetic polyvinylalcohol microspheres," Food Chemistry, vol. 74, no. 3, pp. 281-288, 2001.

[14] L. Campanella, A. Bonanni, E. Finotti, and M. Tomassetti, "Biosensors for determination of total and natural antioxidant capacity of red and white wines: comparison with other spectrophotometric and fluorimetric methods," Biosensors and Bioelectronics, vol. 19, no. 7, pp. 641-651, 2004.

[15] A. Luximon-Ramma, T. Bahorun, A. Crozier, V. Zbarsky, K. P. Datla, and D. T. Dexter, "Characterization of the antioxidant functions of flavonoids and proanthocyanidins in Mauritian 
black teas," Food Research International, vol. 38, no. 4, pp. $357-$ 367, 2005.

[16] B. Serra, S. Jiménez, M. L. Mena, A. J. Reviejo, and J. Pingarrón, "Composite electrochemical biosensors: a comparison of three different electrode matrices for the construction of amperometric tyrosinase biosensors," Biosensors and Bioelectronics, vol. 17, no. 3, pp. 217-226, 2002.

[17] Y.-C. Tsai and C.-C. Chiu, "Amperometric biosensors based on multiwalled carbon nanotube-Nafion-tyrosinase nanobiocomposites for the determination of phenolic compounds," Sensors and Actuators B, vol. 125, no. 1, pp. 10-16, 2007.

[18] J. C. Espin, R. Varon, L. G. Fenoll, et al., "Kinetic characterization of the substrate specificity and mechanism of mushroom tyrosinase," European Journal of Biochemistry, vol. 22, pp. 1270-1279, 2000. 

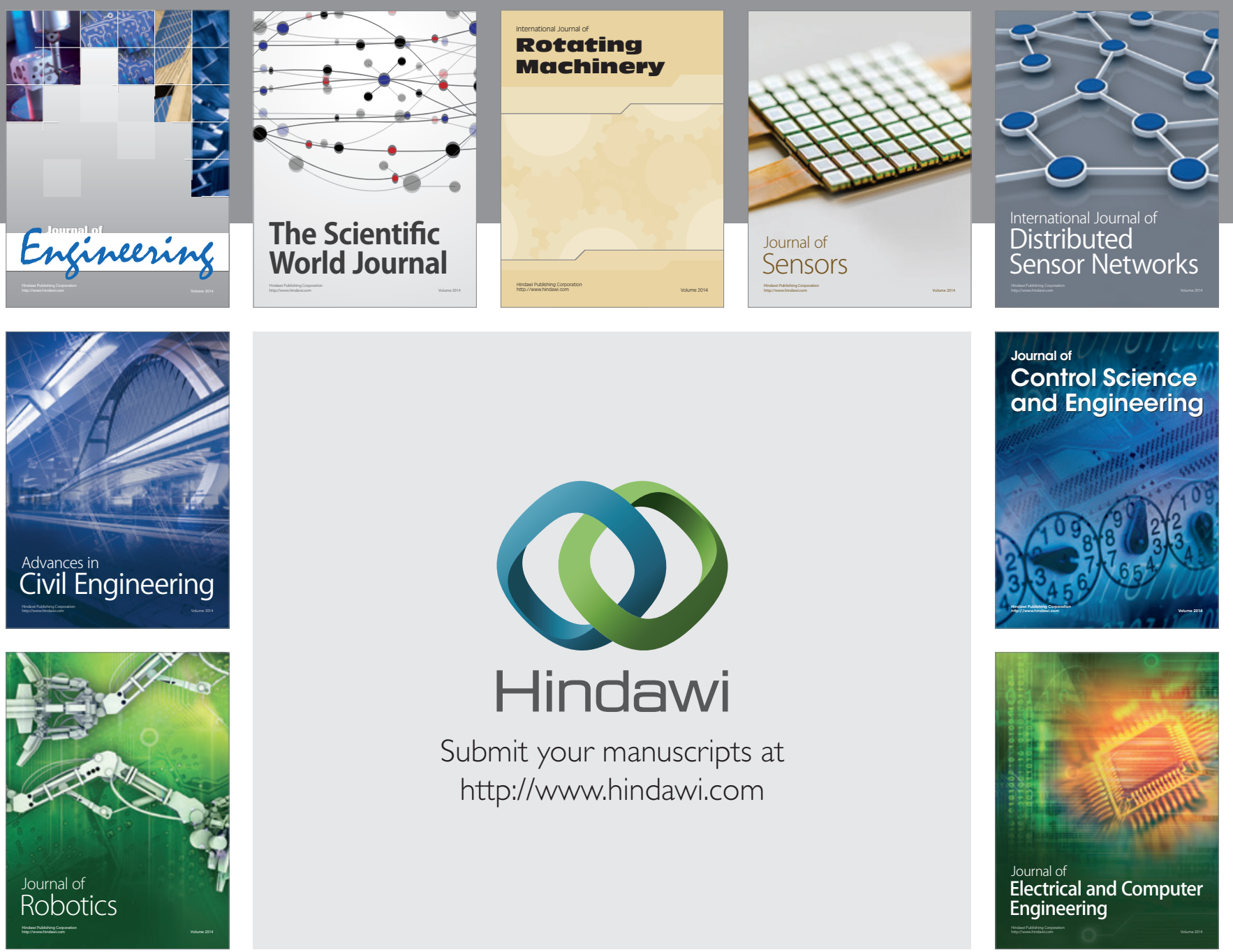

Submit your manuscripts at

http://www.hindawi.com
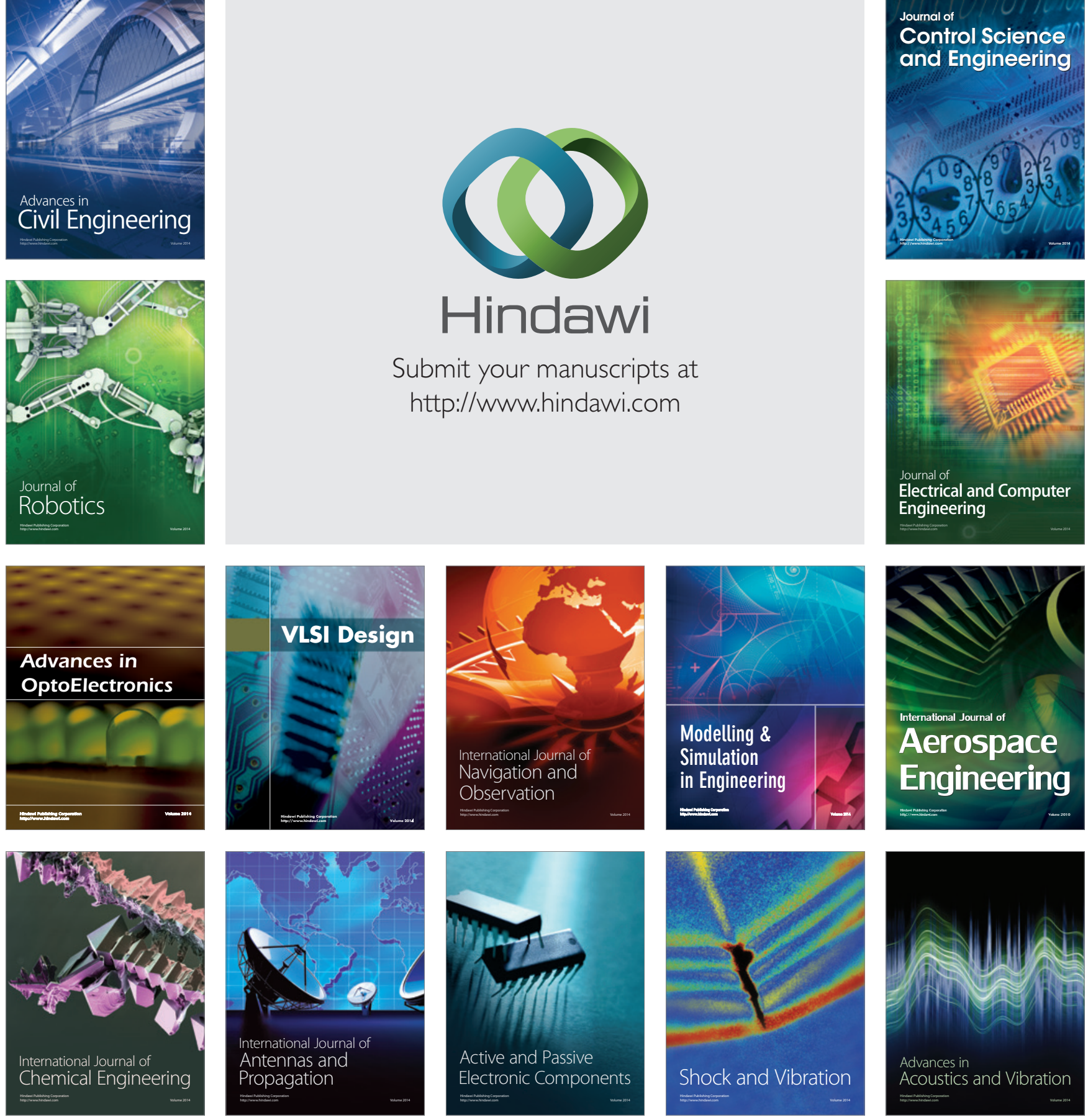